\title{
Novel upper gastrointestinal monitoring system to track upper gastrointestinal bleeding: A pilot study
}

\section{다)(요 $\odot$}

\section{Authors}

Chiao-Hsiung Chuang ${ }^{1,2}$, Chien-Cheng Chen ${ }^{2}$, Jhong-Han Wu ${ }^{1}$, Ming-Yuan Hong ${ }^{3}$, Jui-Wen Kang ${ }^{1}$, Hsin-Yu Kuo ${ }^{1}$, Chien-Jui Huang ${ }^{1}$, Chiung-Yu Chen ${ }^{1}$

\section{Institutions}

1 Department of Internal Medicine, National Cheng Kung University Hospital, College of Medicine, National Cheng Kung University, Tainan, Taiwan

2 Medical Device Innovation Center, National Cheng Kung University, Tainan, Taiwan

3 Department of Emergency Medicine, National Cheng Kung University Hospital, College of Medicine, National Cheng Kung University, Tainan, Taiwan

submitted 7.6.2020

accepted after revision 20.8.2020

\section{Bibliography}

Endoscopy International Open 2020; 08: E1811-E1816

DOI 10.1055/a-1266-3501

ISSN 2364-3722

(c) 2020. The Author(s).

This is an open access article published by Thieme under the terms of the Creative Commons Attribution-NonDerivative-NonCommercial License, permitting copying and reproduction so long as the original work is given appropriate credit. Contents may not be used for commecial purposes, or adapted, remixed, transformed or built upon. (https://creativecommons.org/licenses/by-nc-nd/4.0/)

Corresponding author

Chiung-Yu Chen, Department of Internal Medicine, National Cheng Kung University Hospital, College of Medicine, National Cheng Kung University, 138 Sheng-Li Road, Tainan, Taiwan
Fax: +1-886-62766116

chiungyu@mail.ncku.edu.tw

\section{ABSTRACT}

Background and study aims Early detection of upper gastrointestinal (UGI) rebleeding is not easy by observing clinical symptoms. We developed a novel UGI monitoring system and aimed to test its feasibility of continuous tracking of UGI bleeding.

Patients and methods A prospective study was conducted on patients with moderate to high risk of rebleeding. The UGI monitoring system was installed to monitor their gastric contents. It would alarm if rebleeding was suspected and the physician could review the images to make a further decision. The patient's comfort level was also evaluated.

Results Sixteen patients were enrolled. Rebleeding occurred in one patient and was detected by this system more than 5 hours earlier than with clinical symptoms. The interobserver reliability for reviewing the images to define the blood clearance in the stomach was excellent (intraclass correlation coefficient $0.79-0.96)$. The comfort level assessed by patients was $1.90 \pm 1.39$ (on the scale of $0-5$ ).

Conclusions This pilot study demonstrated the potential of this UGI monitoring system for early detection of rebleeding.

\section{Introduction}

Acute upper gastrointestinal (UGI) bleeding is common and can be life-threatening, particularly in older individuals [1-3]. Despite progress with endoscopic hemostatic techniques, rebleeding still occurs in $13 \%$ to $20 \%$ of patients after primary endoscopy $[1,4]$. Thus, high-risk patients have to be hospitalized for close monitoring and repeat endoscopy is inevitable if massive rebleeding occurs.

Current ways of detecting rebleeding mainly depend on monitoring hemodynamic instabilities and the occurrence of hematemesis, hematochezia, or persistent melena [5, 6]. However, at least a $15 \%$ to $30 \%$ loss of blood volume is required to signicantly influence blood pressure and heart rate [6, 7]. Furthermore, despite the persistent melena is an important symptom of UGI rebleeding $[7,8]$, it is often difficult to determine whether melena is caused by rebleeding or by old blood stasis in the bowel. Therefore, assessing the need for or timing of repeated endoscopy is challenging. Moreover, such uncertainty would cast doubt and delay on diagnosing rebleeding, cause anxiety, and add care burden.

In this study, we developed a UGI monitoring system for continuous tracking of UGI rebleeding and aimed to test its feasibility. This monitoring system was capable of taking images from the stomach regularly and simultaneously analyzing them for the presence of blood. 


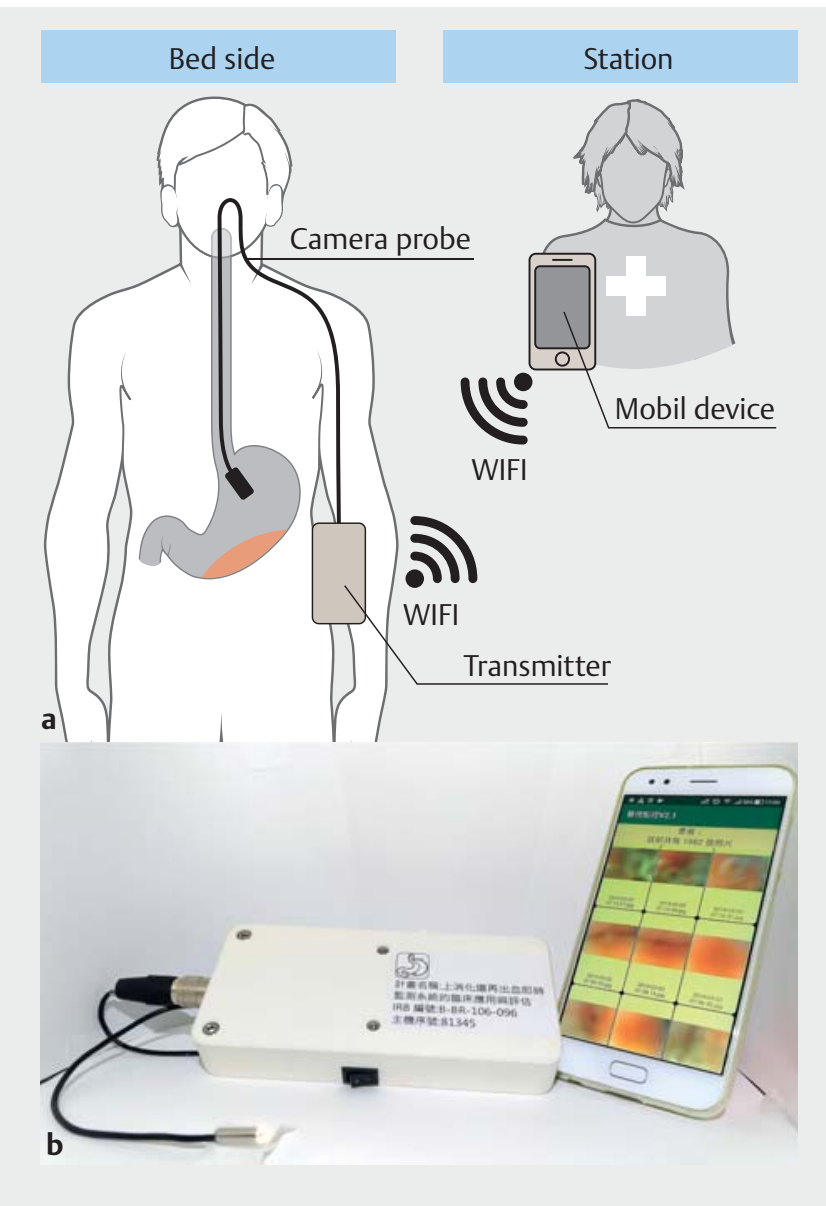

- Fig. 1 Schematic diagram and image of the UGI monitoring system. a The camera probe is placed in the patient's stomach and the transmitter regularly sends images to the mobile device. The mobile device can analyze the images for bleeding detection. $\mathbf{b}$ The black cable with a stainless-steel tubular sensor is the indwelling camera probe. The white box is the transmitter. The mobile device with the SBI application is shown on the right side.
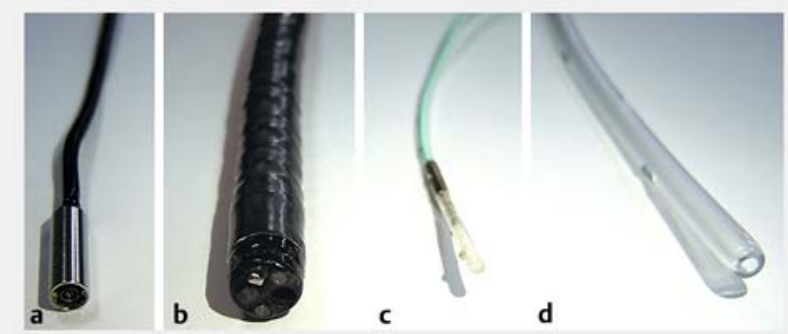

- Fig. 2 Comparison of equipment diameters. a Custom-made camera probe of the UGI monitoring system, the size of front tubular sensor housing: diameter $5 \mathrm{~mm}$, length $15 \mathrm{~mm}$; black cable: diameter $2.3 \mathrm{~mm}$, b gastroscope: diameter $9.9 \mathrm{~mm}$, c esophagus pH meter: diameter $2.0 \mathrm{~mm}$, and $\mathbf{d} 16$ French NG tube: diameter $5.3 \mathrm{~mm}$.

\section{Patients and methods}

We performed a pilot study of the UGI monitoring system. This study was approved by the Institutional Review Board at $\mathrm{Na}$ tional Cheng Kung University Hospital and the Taiwan Food and Drug Administration. Patients were enrolled for the study from October 12, 2018 to October 28, 2019.

\section{Design of UGI monitoring system}

The UGI monitoring system consists of a camera probe, a wearable transmitter, and a mobile device with an application for analyzing suspicious bleeding indicators (SBI). A schematic plot and a picture of the system are shown in $>$ Fig. 1. Technical specifications of the camera are $640 \times 480$ resolution, $90^{\circ}$ field of view, and $15-\mathrm{mm}$ focus distance. The light sources are four light-emitting diodes embedded around the camera. Besides regularly taking pictures in the stomach, the device can be switched to a real-time video mode. Usually, the real-time mode is used during the camera probe insertion and the physician canse use real-time imaging during insertion from the nose to the stomach. The real-time mode also can be used to reconfirm rebleeding when the system sets off an alarm. Furthermore, - Fig. 2 shows a comparison of the diameters of the camera probe, nasogastric tube, esophagus $\mathrm{pH}$ meter, and a UGI endoscope. The black cable diameter is $2.3 \mathrm{~mm}$ and the front tubular sensor housing is $5 \mathrm{~mm}$ in diameter and $15 \mathrm{~mm}$ long.

The SBI application was used to set a bleeding threshold using the ratio of red/green color intensities. The red/green color intensities were obtained by totaling all pixel values of red or green color in the acquired images. The red/green ratio was calculated by dividing the red color intensity with the green one. In this study, the bleeding threshold was set at 2.0. If the calculated ratio was higher than 2.0 , the mobile device would alarm.

\section{Patient enrollment and study design}

Patients older than 20 years were eligible for this study. All the enrolled patients were assessed as moderate to high risk of rebleeding (Rockall score $\geq 3$ ) after undergoing therapeutic UGI endoscopy. Exclusion criteria included having an NG tube placement in the patient and inability to pass the camera probe through the nostrils.

The UGI monitoring system was installed at the bedside and left in until the attending physicians decided to stop or a patient was unwilling to continue (a maximum of 3 days). The camera probe was inserted under direct vision in the real-time mode and was placed in the stomach about 10 to $20 \mathrm{~cm}$ below the esophagogastric junction. In this study, clinical management, such as timing of second-look endoscopy or oral intake, was held to the standard as much as possible. However, attending physicians could use the UGI monitoring system as a tool to help with clinical management. The enrolled patients were asked not to ingest any red liquid so as to avoid confusion with blood. 


\section{Outcome measures}

The primary outcome was time to detection of rebleeding determined by our system, compared with clinical rebleeding. The UGI monitoring system triggered an alarm for suspicion of rebleeding and physicians could review images from it for decision-making. Clinical rebleeding was defined as vomiting of fresh blood or coffee grounds, hematochezia or melena after a normal stool, a drop in hemoglobin level of more than $2 \mathrm{~g} / \mathrm{dL}$, or blood in the stomach on second-look endoscopy $[9,10]$.

The secondary outcomes were patient comfort level, interobserver agreement in image interpretation, and timing of blood clearance in the stomach. Patient comfort level was evaluated by using a Likert scale to score from 0 for no discomfort to 5 for extreme discomfort. Time of blood clearance in our system was determined by experienced endoscopists when no more blood or coffee grounds were visible in the gastric images. The disappearance of melena or bloody stool was determined by an experienced nurse and compared with that determined by using our system. During system operation, an incharge endoscopist responded to alarms, checked blood clearance time, and made decisions. After the study, another two independent endoscopists retrospectively reviewed the series of images to determine the rebleeding and blood clearance time. The interrater reliability of determining the time of gastric blood clearance among these three endoscopists was assessed by intraclass correlation coefficient analysis.

\section{Results}

Nineteen patients were screened and invited to particpate. Two refused to sign the informed consent and one declined to continue on the trial during insertion of the camera probe, thus, data from 16 patients were analyzed. ThelR baseline characteristics were summarized in $>$ Table 1 . After introducing our device, describing procedures for the clinical trial, and obtaining informed consent of patients or their families, the system was set in a mean of 4.5 hours after index endoscopy (range 1-12 hours). - Fig. 3 shows images of various gastric contents taken by our UGI monitoring system. Although the images are blurry, the three experienced endoscopists were able to identify the condition in the stomach and determine timing of rebleeding or blood clearance by reviewing the color changes along the elapsed time.

An 81-year-old male patient with Forrest Ila duodenal ulcer had rebleeding. $\mathbf{F i g . 4}$ shows the serial changes in red/green ratios and all events that occurred during monitoring. In this patient, the SBI analysis alarmed more than 5 hours earlier than endoscopy confimed the clinical diagnosis of rebleeding. The initial red/green ratios were low when the patient's stomach was empty. The ratios increased when fresh blood appeared in the stomach and fell after the blood was expelled. Three endoscopists reviewed the serial images and estimated the time point of rebleeding at around 2:30 to 2:42 am on Day 1 , which was quite consistent with the first time of system alarm, which was 2:33 am. Except for this patient, the rest of the enrolled patients had no rebleeding, but in four patients,
- Table 1 Baseline characteristics and endoscopic findings for 16 enrolled patients.

\begin{tabular}{|c|c|}
\hline Clinical characteristics & $\begin{array}{l}\text { Number or mean } \\
\text { (range) }\end{array}$ \\
\hline Age (years) & $66.7(41-84)$ \\
\hline Sex (female/male) & $3 / 13$ \\
\hline \multicolumn{2}{|l|}{ Clinical presentations } \\
\hline - Coffee ground/fresh blood vomitus & $8 / 3$ \\
\hline - Tarry stool/tarry-bloody stool & $9 / 3$ \\
\hline - Abdominal pain & 4 \\
\hline \multicolumn{2}{|l|}{ Baseline comorbidities } \\
\hline - Chronic kidney disease & 7 \\
\hline - Coronary arterial disease & 1 \\
\hline - Chronic liver disease & 3 \\
\hline - Diabetes & 2 \\
\hline - Previous stroke & 1 \\
\hline \multicolumn{2}{|l|}{ Causes of bleeding } \\
\hline - Gastric ulcer & 9 \\
\hline - Duodenal ulcer & 6 \\
\hline - Gastric cancer & 1 \\
\hline Rockall score & $6.75(3-9)$ \\
\hline \multicolumn{2}{|l|}{ Forrest classification } \\
\hline . la/lb & $1 / 4$ \\
\hline - Ila/IIb/llc & $8 / 2 / 1$ \\
\hline . III & 0 \\
\hline \multicolumn{2}{|l|}{ Endoscopic therapy ${ }^{1}$} \\
\hline $\begin{array}{l}\text { " Diluted epinephrine submucosal injec- } \\
\text { tion }\end{array}$ & 11 \\
\hline - Argon plasma/bipolar coagulation & $1 / 6$ \\
\hline - Hemoclipping/rubber band ligation & $8 / 1$ \\
\hline
\end{tabular}

the SBI alarm was triggered. In two cases, it was induced by residual fresh blood in the stomach, in one, by drinking grape juice, and in one by hemorrhagic gastritis.

For the secondary outcomes, most patients tolerated the camera probe well, and the mean comfort level was $1.90 \pm$ 1.39. Interobserver reliability could not be determined because only one patient had rebleeding. Instead, the time of gastric blood clearance determined by three endoscopists was used for the analysis of the inter-observer agreement. The interrater reliability was excellent with an intraclass correlation coefficient of 0.90 (95\% confidence interval $0.79-0.96$ ). The mean time of blood clearance determined by our system was 10.6 hours after the index endoscopy. That was much earlier than the mean time of clinical disappearance of melena or bloody 

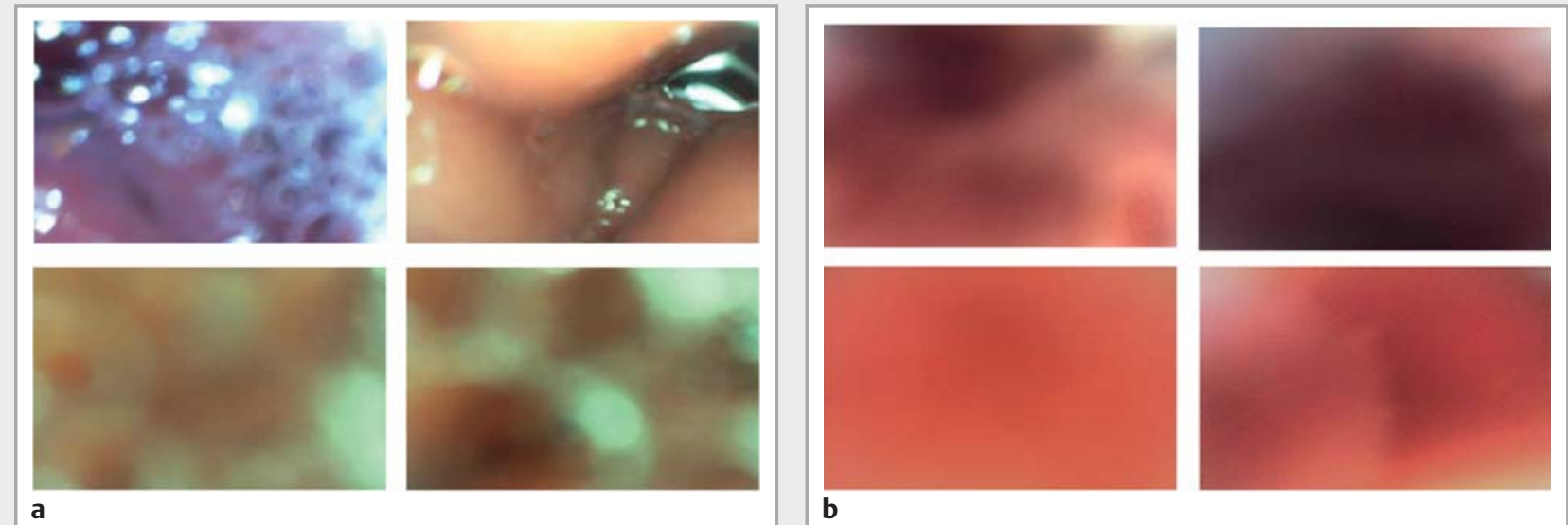

a

\section{b}
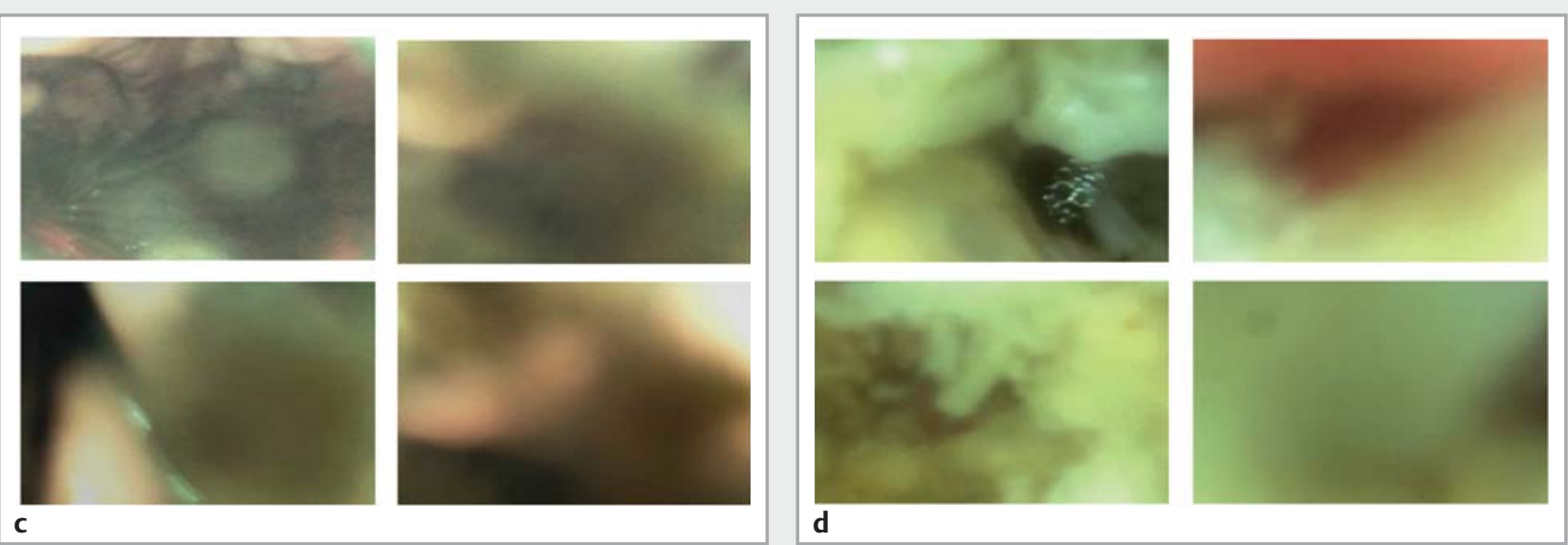

Fig. 3 Images taken by the UGI monitoring system. a The images showed that there were only bubbles in the stomach, indicating that blood had been cleared from the stomach. $\mathbf{b}$ The brisk red-colored images indicate the presence of fresh blood in the stomach. $\mathbf{c}$ The system showed dark-colored images when there were coffee-ground or dark blood clots in the stomach. $\mathbf{d}$ The images showed food in the stomach.

stool, which was 97.9 hours after index endoscopy ( $\triangleright$ Table 2 ). No serious adverse events occurred.

\section{Discussion}

In this study, we developed a UGI monitoring system for tracking patients with UGI bleeding. To our knowledge, it is the first system in the world designed for this purpose. Using continuous and real-time monitoring with the SBI alarm application, the UGI monitoring system could detect rebleeding earlier than the conventional methods. This information may be helpful to make a precise treatment plan, such as to hold oral intake or to do a second-look endoscopy. Thus, it may increase medical efficiency, reduce complications, and save costs.

The correlation was excellent among the three endoscopists regarding determination of time of rebleeding or blood clearance in the stomach. It demonstrates that using eye recognition to differentiate color changes in serial images of our system is easy and accurate. Our strategy to analyze red/green ratios of an image as the threshold of SBI alarm was successful as seen in our rebleeding patient, the red/green ratio rose and the $\mathrm{SBI}$ application alarmed when rebleeding occurred. The time of rebleeding either determined by eye recognition or by system automatic analysis was quite close within 12 minutes. By realtime monitoring in the gastric contents, our UGI monitoring system could detect rebleeding earlier than the conventional methods by observation of clinical parameters or stool color changes. Nevertheless, the current guidelines recommend endoscopy within 24 hours for UGI bleeding [11]. Besides, a recent study demonstrates that urgent endoscopy within 6 hours for the patients with Glasgow-Blatchford score of 12 or higher didn't improve 30 days mortality [12]. Accordingly, finding patients that will be benefited by detecting "early" rebleeding by our system will require further studies to explore.

Moreover, resuming normal stool color is commonly used as an indicator of "bleeding cessation". However, the melena may persist for days by old blood stasis in the bowel. In this study, we found the system determined gastric blood clearance time was always earlier than that judged by the disappearance of melena or bloody stool. Therefore, our system may relieve unnecessary anxiety for observing patients with persistent black stool and reduce clinical care burden.

Although the outer diameter of the front tubular sensor housing of our camera probe was $5 \mathrm{~mm}$, the black cable was 


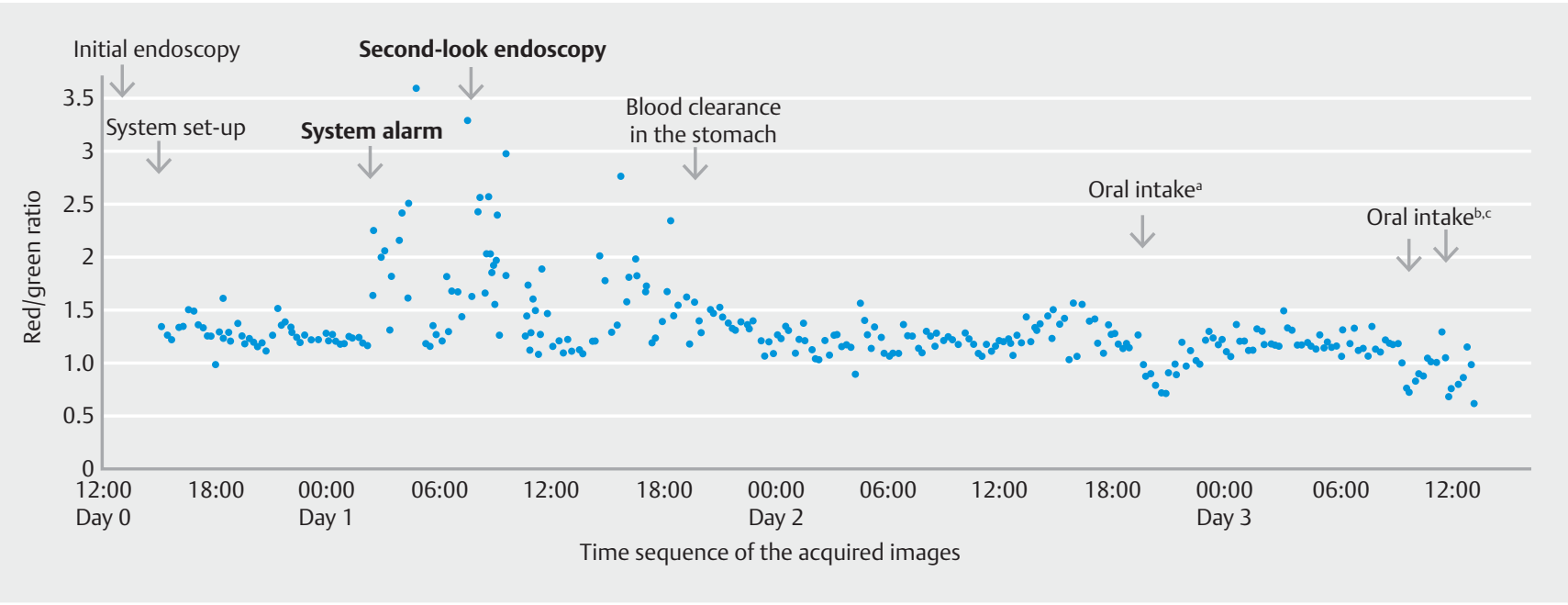

- Fig. 4 Red/green ratio plots from a patient with rebleeding during the monitoring. The system was set up about 2 hours after the index endoscopy. It alarmed at 2:33 am on Day 1 and the red/green ratios rose to more than 1.5 or even 2.0 after that timepoint. The patient had stable vital signs and no discomfort; endoscopy did not proceed until 8:00 am. The second-look endoscopy showed a Forrest lb ulcer that was treated with rubber band ligation. The average time of blood clearance in the stomach estimated by three endoscopists was at 20:12 on Day 1 . After that time, the red/green values returned to less than 1.5. Besides, after oral intake (A drug pills, C $100 \mathrm{~mL}$ Ensure). The red/green ratios had transient drops.

- Table 2 The time lag of the bleeding cessation estimated by our UGI monitoring system or observation of stool color change.

\begin{tabular}{|l|c|}
\hline \begin{tabular}{l} 
Timepoint of the index endoscopy (t0) \\
\hline Timepoint to set up our system (hours after t0)
\end{tabular} & Mean (range) \\
\hline $\begin{array}{l}\text { Timepoint of blood clearance in the stomach } \\
\text { estimated by using our system (hours after t0) }\end{array}$ & $10.6(1-35)$ \\
\hline - Observer 1 & $11.3(1-34)$ \\
\hline - Observer 2 & $10.3(1-35)$ \\
\hline - Observer 3 & $10.1(1-34)$ \\
\hline $\begin{array}{l}\text { Timepoint of the disappearance of melena or } \\
\text { bloody stool (hours after t0) }\end{array}$ & $97.9(5-171)$ \\
\hline $\begin{array}{l}\text { The time lag between these two methods (hours) } \\
\text { UGl, upper gastrointestinal. }\end{array}$ & $87.3(4-169)$ \\
\hline $\begin{array}{l}\text { The blood clearance time estimated by each observer correlated signifi- } \\
\text { cantly with those of the other two observers (intraclass correlation coeffi- } \\
\text { cient 0.79-0.96). }\end{array}$
\end{tabular}

only $2.3 \mathrm{~mm}$. Thus, most of the enrolled patients were able to tolerate the discomfort of inserting and wearing the camera probe. Previous studies have discussed the comfort level of wearing devices similar to our camera probes, such as NG tube and esophageal $\mathrm{pH}$ monitor $[13,14]$. Our study shows that the comfort level of the UGI monitoring system was similar to that in a previous esophageal $\mathrm{pH}$ monitoring study $(1.90 \pm 1.39$ vs. $2.11 \pm 1.16$ ) [14]. IN addition, most of the enrolled patients ate during the monitoring period, and that interfered with the intercamera probe in only a few cases. As technology advances, the size of the tubular sensor and cable could be further decreased to improve patient comfort.
Application of this system also could be extended to the emergency department (ED) for assessing emergent endoscopic hemostasis. Some studies have shown that capsule endoscopy has the potential to be used to triage patients with UGI bleeding who do not require admission, which would reduce hospital burden $[15,16]$. Schmidt et al also reported that detection of acute UGI bleeding with a non-imaging sensor capsule is feasible and safe [17]. Thus, it could be worth conducting a study using our system for risk stratification and monitoring in an ED setting before endoscopy.

Because the camera sensor hangs in the stomach, it raises a concern that duodenal ulcer rebleeding may have not result in sufficient blood flow back to the stomach to trigger the alarm. A limited number of cases duodenal ulcer bleeding were included in this study; therefore, further study is needed to verify the sensitivity of the rebleeding alarm in that setting. Nevertheless, in our clinical experiences if the bleeder is located at the bulb, as in the patient in the present study, the duodenal blood can flow back into the stomach. Ogasawara et al. also showed that most bleeding duodenal ulcers are located at the bulb rather than in the second portion (106 vs. 17 patients) [18].

Our study have some limitations. First, the case number was small because this was a first-in-human study for this novel system. Gvien thatonly one case of rebleeding occurred in this pilot trial, the patient selection criteria may need to be revised in future trials to include high-risk patients, such as Forrest I or possibly lla. Second, although patient comfort with our system is similar to that in a previous study of a 24-hour pH meter [14], there were still two patients who could not tolerate and discontinued the study. Thus, the surface material and diameter of the camera probe need to be improved. Third, there weree two false alarms caused by drinking grape juice and hemorrhagic gastritis, which would result in additional demand on a care-gi- 
ver's time. That problem can be partially solved by instructing patients not to ingest any red liquid, reviewing the serial uploaded images, or checking the real-time video. Moreover, further precise imaging analysis will be developed to avoid false alarm.

\section{Conclusion}

In conclusion, this monitoring system is the first device intended to continuously track bleeding in the UGI tract. This smallscale pilot study demonstrated the potential of the system for early detection of UGI rebleeding and determination of bleeding cessation. The interobserver reliability for interpretation of the images was excellent.

\section{Acknowledgement}

The study was supported by a research grant from the Ministry of Health and Welfare (MOHW108-TDU-B-211-133003 and MOHW109-TDU-B-211-114003).

\section{Competing interests}

The authors declare that they have no conflict of interest.

\section{References}

[1] Louis HSL, Sung J]. Treatment of UGI bleeding in 2020: new techniques and outcomes. Dig Endosc 2020: doi:10.1111/den.13674

[2] Sengupta N, Tapper EB, Patwardhan VR et al. High Glasgow Blatchford Score at admission is associated with recurrent bleeding after discharge for patients hospitalized with upper gastrointestinal bleeding. Endoscopy 2016; 48: 9-15

[3] Barkun AN, Bardou M, Kuipers E] et al. International consensus recommendations on the management of patients with nonvariceal upper gastrointestinal bleeding. Ann Intern Med 2010; 152: 101-113

[4] Mangiafico S, Pigò F, Bertani $\mathrm{H}$ et al. Over-the-scope clip vs epinephrine with clip for first-line hemostasis in non-variceal upper gastrointestinal bleeding: a propensity score match analysis. Endosc Int Open 2020; 8: E50-E58
[5] Nam JH, Jeon TJ, Cho JH et al. Risk factors of the rebleeding according to the patterns of nonvariceal upper gastrointestinal bleeding. Turk J Gastroenterol 2017; 28: 266-271

[6] Maggio D, Barkun AN, Martel M et al. Reason Investigators. Predictors of early rebleeding after endoscopic therapy in patients with nonvariceal upper gastrointestinal bleeding secondary to high-risk lesions. Can J Gastroenterol 2013; 27: 454-458

[7] Sheibani S, Kim JJ, Chen B et al. Natural history of acute upper Gl bleeding due to tumors: short-term success and long-term recurrence with or without endoscopic therapy. Aliment Pharmacol Ther 2013; 38: 144-150

[8] Hong M], Lee SY, Kim JH et al. Rebleeding after initial endoscopic hemostasis in peptic ulcer disease. J Korean Med Sci 2014; 29: 1411 1415

[9] Cheng HC, Yang EH, Wu CT et al. Hypoalbuminemia is a predictor of mortality and rebleeding in peptic ulcer bleeding under proton pump inhibitor Use. J Formos Med Assoc 2018; 117: 316-325

[10] Sung J], Barkun A, Kuipers EJ et al. Intravenous esomeprazole for prevention of recurrent peptic ulcer bleeding: a randomized trial. Ann Intern Med 2009; 150: 455-464

[11] Laine L, Jensen DM. Management of patients with ulcer bleeding. Am J Gastroenterol 2012; 107: 345-361

[12] Lau JYW, Yu Y, Tang RSY et al. Timing of endoscopy for acute upper gastrointestinal bleeding. N Engl J Med 2020; 382: 1299-1308

[13] Singer AJ, Richman PB, Kowalska A et al. Comparison of patient and practitioner assessments of pain from commonly performed emergency department procedures. Ann Emerg Med 1999; 33: 652-658

[14] Musser J, Kelchner L, Neils-Strunjas J et al. Patient Compliance during 24-Hour Dual pH Probe Monitoring for Extraesophageal Reflux. ISRN Otolaryngol 2011: 703936

[15] Sung J], Tang RS, Ching JY et al. Use of capsule endoscopy in the emergency department as a triage of patients with GI bleeding. Gastrointest Endosc 2016; 84: 907-913

[16] Shah N, Chen C, Montano N et al. Video capsule endoscopy for upper gastrointestinal hemorrhage in the emergency department: A systematic review and meta-analysis. Am J Emerg Med 2020; 38: 12451252

[17] Schmidt A, Zimmermann M, Bauder M et al. Novel telemetric sensor capsule for EGD urgency triage: a feasibility study. Endosc Int Open 2019; 7: E774-E781

[18] Ogasawara N, Mizuno M, Masui R et al. Predictive factors for intractability to endoscopic hemostasis in the treatment of bleeding gastroduodenal peptic ulcers in Japanese patients. Clin Endosc 2014; 47: $162-173$ 